\title{
Comparison between two Different Splints Used for Fixation of Symphyseal and Parasymphyseal Mandibular Fractures in Pediatric Infants
}

\author{
Yomna Elshazly \\ Department of Prosthodontics, Faculty of Dentistry, Alexandria University, Egypt
}

\begin{abstract}
Mandibular fractures in the pediatric population are uncommon. The conservative treatment is in most cases advocated before the use of internal rigid fixation because they have a negative effect on skeletal growth and unerupted teeth. This paper presents two groups, group A treated by fenestrated acrylic splintand group $B$ treated by modified oropharyngeal airway tube to be used as a splint,both are constructed on the mandibular stone casts and fixed in place with circummandibular wires. Data was collected and analysed to compare between the two types of splints. The fenestrated splint showed better results than the modified airway tube.
\end{abstract}

Keywords: Trauma, mandible, fracture, children and splints

\section{Introduction}

The maxillofacial trauma is common in the world and the mandible is a common bone to fracture in the pediatric facial trauma. During the first years of life, the size and proportions of the facial skeleton change markedly. The facial skeleton increases in relation to the rest of the head, and the sinuses and dentition develop postnatally. The mandible is relatively small at birth and grows by remodeling.

McLennan 1956 reported that 1\% of mandibular fractures occurred in children younger than 6 years [1]. Rowe's 1969 stated $1 \%$ occurred in patients younger than 5 years [2]. Thoren 1992 described that only 1 to $2 \%$ mandibular fractures occur in patients younger than 6 years[3]. Some authors reported an equal distribution between the gender and others describe 2:1 male predominance for pediatric mandibular fractures [1,4-6].

Children of one year of age, fractures of the mandible are rare whereas in older children common causes of maxillofacial fractures are; road traffic accidents (RTA), falls, sports activities, violence and abuse assaults[5,6,7].

Young patients are more difficult for evaluating occlusion and condyles. The step deformity and instability of jaw opening may be the only apparent signs of the fracture of the mandible in these children, further confirmation and characterization of the fracture type, location and pattern should be performed with radiographic imaging.

The successful management of fractures should result in restoration of occlusion and facial balance. Usually, the deciduous teeth are not completely erupted before the age of 2 years and the children at this stage of development are treated as though edentulous and, open reduction and fixation with mono-cortical screws at the inferior border of the mandible is limited to comminuted and unfavourable types of mandibular fractures to avoid damaging the underlying developing teeth $[4,8,9]$.
The pediatric mandibular trauma up to age 2 years had been reported and we treated these patients, using two different management modalities.

The purpose of this study was to highlight and treat these extremely uncommon fractures which are usually unnoticed by the parents or general practitioners with minimal postoperative complications and to compare between the two types of splints used in mandibular fracture reduction and fixation.

\section{Literature Survey}

The authors bring to our attention the epidemiology ofmandibular fractures that occur in infancy. An infant whofalls from even a limited height and sustains chin regionabrasions or lip laceration and swelling and ecchymoses of the floor of the mouth is at risk for a minimally displacedsymphyseal fracture of the mandible. Unless specific radiographicdocumentation is obtained, their fractures are likelyto be missed. Fortunately, the authors have demonstratedthat surgical reduction and immobilization is rarely requiredto achieve adequate healing.

Lustmann and Milhem's [10] report reminds us of the evolvinganatomy of the mandible through growth and development.

During infancy the thick, short condylar neck is able to withstandsignificant force, with only a limited number of contrecoup fractures occurring in combination with parasymphyseal fractures. This fracture pattern changes as thecondylar neck thins, becoming tall and cortical later in childhood.The maxillofacial surgeon called on to evaluate the traumatizedinfant must still apply general principles:[7]

1) Recognizeother organ system injuries;

2) Examinethe entire craniomaxillofacial complex;

3) Obtain radiographs necessaryto confirm or refute clinical impressions;

4) Treat significantlydisplaced and mobile fractures; 


\section{International Journal of Science and Research (IJSR) \\ ISSN (Online): 2319-7064 \\ Index Copernicus Value (2013): 6.14 | Impact Factor (2015): 6.391}

5) Consider all treatment options and then select the one most appropriate for the clinicalsituation; and

6) Institute long-term patient follow-up throughgrowth and development.

When an infant or young child does present with a mandibular fracture that is displaced and mobile, it should be reduced and fixed either with an acrylic splint and circumandibular wires; arch bars and maxillomandibular fixation (depending on the extent of the primary dentition) it should be avoided to prevent ankylosis; or open reduction with a miniplate or microplate and screw fixation along the inferior border of the mandible with caution to avoid injury of teeth buds [11]. The use of biodegradable systems to avoid the need for a second surgery to remove the hardwears[12]. In a patient with multiple organ system injury, including crania-orbital injuries, brain concussion, and a displaced parasymphyseal fracture of the mandible, it may be most expedient to manage the parasymphyseal fracture with open reduction and microplate stabilization to avoid the "need for tracheostomy [13].

\section{Methods}

This study consists of 14 infants under the age of 2 years divided into two groups each group contains 6 infants:

1. Group 1 consists of 7 infants will be treated with the fenestrated splints.

2. Group 2 consists of 7 infants will be treated by the modified oropharyngeal airway tube to be used as a splint.

\section{Patient selection criteria}

All patients participating in this study will be chosen according to the following criteria:

\section{Inclusion criteria}

Pediatric patients less than 2 years of age with symphyseal or parasymphyseal mandibular fractures with incomplete eruption of deciduous teeth.

\section{Exclusion criteria}

- Brain injuries.

- Complete eruption of deciduous teeth.

- Multiple or comminuted fractures.

Including each infant to group A or group B is done alternately.

\section{Group A: Treated with the fenestrated splint:}

The infant reported to the emergency department Alexandria University with history of falling. History of the trauma is described by the parents of the infant; the patient did not lose consciousness, was well-oriented and had no history of convulsions or vomiting to exclude brain injury. Gaining cooperation for the physical examination can be difficult, especially with children shortly after experiencing a traumatic injury. Gentle examination with encouragement from the child's parents is usually sufficient.

Intraoral examinations revealed step deformity in the occlusion (figure 1), laceration in the gingiva and chin.Complete history of the fall is taken from the parents then the infant is referred to an imaging center for a PA skull $\mathrm{x}$-ray to visualize the fracture exact location and to confirm the diagnosis figure (2).

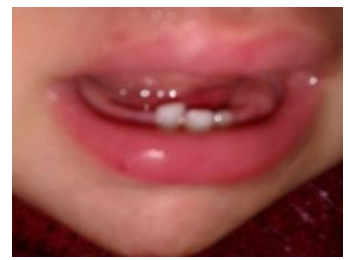

Figure 1: Step deformity

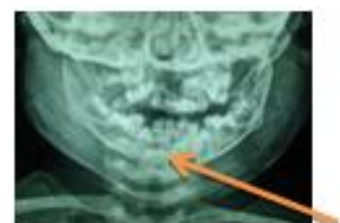

Figure 2: PA skull x-ray showing symphy seal fracture

- Impressions of maxillary and mandibular dental arches figure (3) are taken by fast set alginate irreversible hydrocolloid impression material

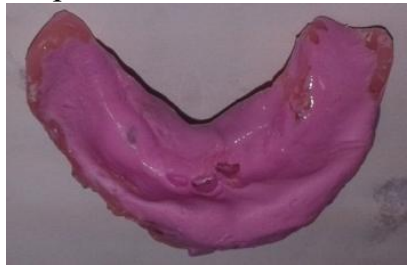

Figure 4: mandibular impression

- On the mandibular cast, the fracture line is marked with the aid of the plain x-ray (PA Skull) and consultation with the surgeon then the cast is cut at the fracture line.

- Mock surgery which simulates the planned surgery will be done by sawing the mandibular cast in the fracture line and rejoin the cast in the proper pretraumatic position and proper teeth alignment (figure 5_6).

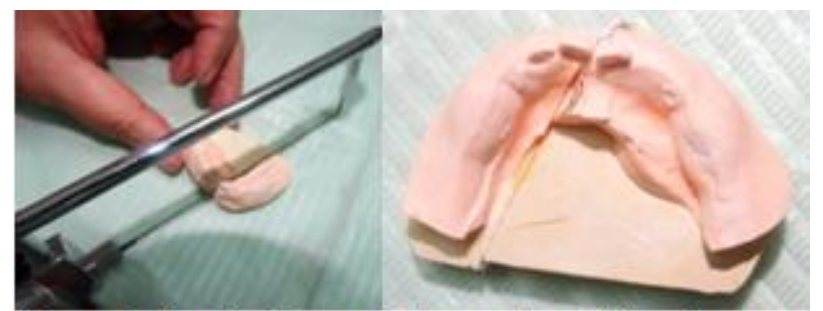

Figure 5: Sawing the cast Figure 6: rejoining the cast

- A notch or a furrow prepared distal to the teeth so that the circum-mandibular wiring can be adapted and fitted in it, the splint must be extended beneath the fracture depth with release of the freni.The fenestrated splint will then be fabricated from the cast using heat cured acrylic resin and its processing as usual. The color of the splint is clear to help show if there is any pressure area on the soft tissue.it should be constructed so that the occlusal surface makes contact with the maxilla or maxillary dentition, and maintains a normal vertical dimension (figure 7). On the buccal or the lingual surface, a reinforcing wire can be incorporated to strength it and avoid its fracture in thin sections. The splint is applied to the lower dental arch and fixation is achieved using two 


\section{International Journal of Science and Research (IJSR) \\ ISSN (Online): 2319-7064}

Index Copernicus Value (2013): 6.14 | Impact Factor (2015): 6.391

to four circummandibularwires the occlusal surface of the teeth should be left open.

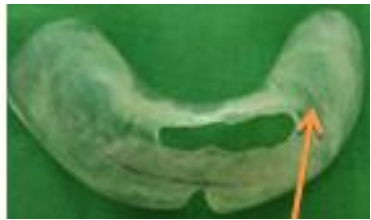

Figure 7: The furrow

- Complete laboratory investigations will be done to make sure that the infant is fitted for general anaethesia and surgery.

\section{Surgical phase:}

The fracture is reduced by gentle manual reduction and fixed with the fenestrated splint, which is secured by wiring around the mandible on each side in the molar region (figure 8-9).
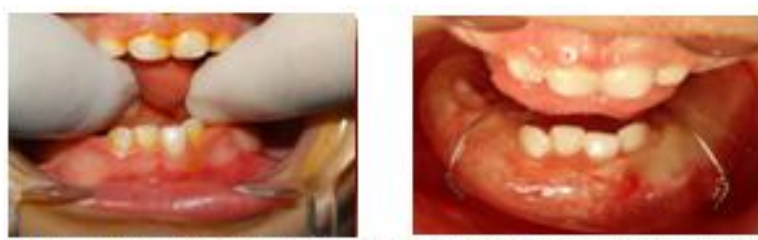

Figure 8: Before reduction Figure 9: After reduction

- Postoperative PA skull $\mathrm{x}$-ray is done to evaluate the fracture reduction (figure 10).

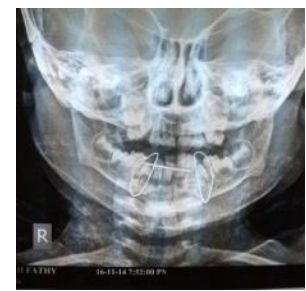

Figure 10: Good fracture reduction and the splint is in place

Patient will be reviewed every week with evaluation of the infant's comfort, oral hygiene, durability of the fenestrated splint and after the fourth week the circum-mandibular wiring and the fenestrated splint will be removed under sedation.

Following removal of the fenestrated splint, a careful examination of the teeth and fracture fragment is carried out to confirm satisfactory healing of the fracture. It is also imperative to monitor such cases over a prolong period so that future eruption of the permanent dentition can be closely observed. Another plain x-ray (Posteroanterior PA) is taken after removal of the fenestrated splint (figure 11).

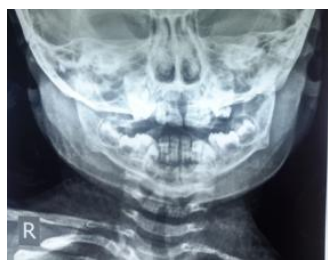

Figure 11: Healing of the fracture line after fixation with the fenestrated splint after 4 weeks
Group B:Treated with the modified oropharyngeal airway tube to be used as a splint [14]:

- Impressions of maxillary and mandibular dental arches are taken by fast set alginate irreversible hydrocolloid impression material using a tray made of wax and fenestrated to hold the alginate (figure 12).

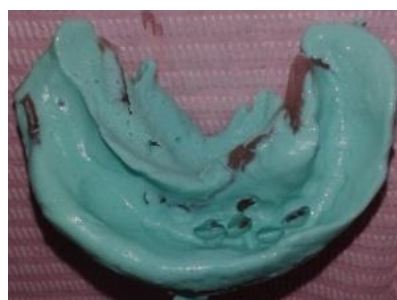

Figure 12: wax tray and mandibular impression.

- Pouring the casts with stone (figure 32).

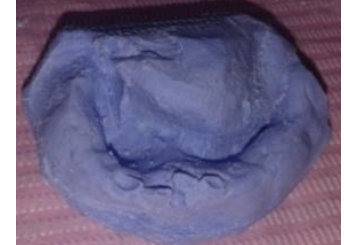

Figure 13: The cast in dental stone

- The proper size of the oropharyngeal airway tube will be chosen to fit the curvature of the mandibular arch (figure 14).

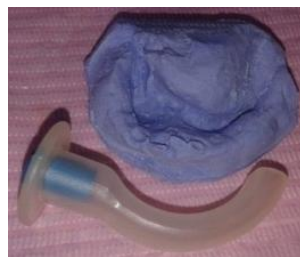

Figure 14: Size of oropharyngeal airway tube corresponding to the mandibular arch

- The guard will be cutted off, then the airway will be splitted longitudinally to leave a longer lingual lip (figure 15-16).

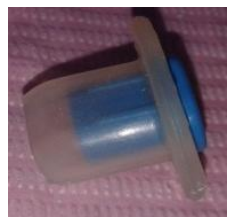

Figure 15: The guard is cutted off

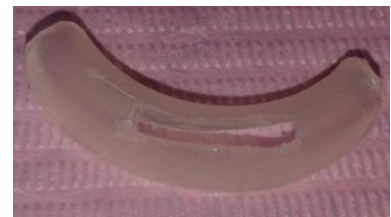

Figure 16: After modifications

- The prepared splint will be fenestrated to accommodate the teeth on the arch (figure 17).

Volume 5 Issue 7, July 2016 www.ijsr.net 


\section{International Journal of Science and Research (IJSR)}

\section{Index C}

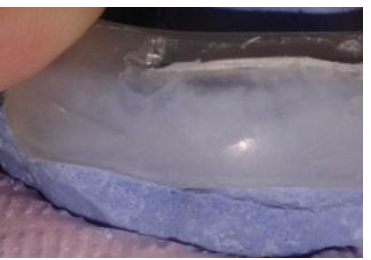

Figure 17: Oropharyngealairway $t$

- This splint made from the airway the lower arch by means of circu.

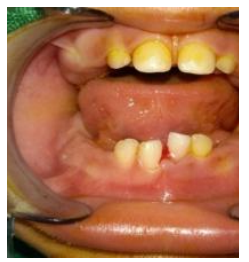

Figure 18: Step de

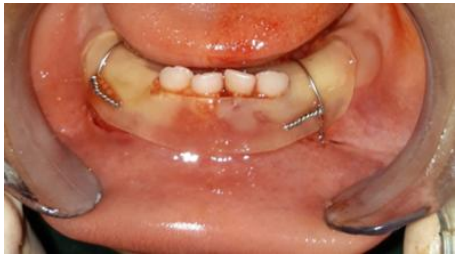

Figure 19: After fixation

\section{Results}

During this study, falling either during playing or from height is the most common cause of mandibular fractures in our study.one case due to road traffic accident and one case due to a horse kick.

No mandibular fracture due to child abuse.

Table 1: Etiology of mandibular fractures in the two groups:

\begin{tabular}{|c|c|c|}
\hline Etiology & Group A & Group B \\
\hline Fall during playing & 2 & 3 \\
\hline Fall from height & 4 & 3 \\
\hline Road Traffic accident & 1 & 0 \\
\hline Horse kick & 0 & 1 \\
\hline
\end{tabular}

Table 2: Location of mandibular fractures in the two groups:

\begin{tabular}{|l|c|c|}
\hline Type of fracture & Group A & Group B \\
\hline Symphysis & 3 & 3 \\
\hline Parasymphysis & 1 & 2 \\
\hline Symphysis+condylar fracture & 1 & 1 \\
\hline Parasymphysis + condylar fracture & 2 & 1 \\
\hline
\end{tabular}

Symphyseal fracture was the most common location among all cases with $42.8 \%$ percentage..

Table 3: Distribution of the studied cases according to sex

\begin{tabular}{|c|c|c|}
\multicolumn{2}{c}{$(\mathrm{n}=14)$} \\
\hline Sex & No. & $\%$ \\
\hline Male & 6 & 42.9 \\
\hline Female & 8 & 57.1 \\
\hline
\end{tabular}

Among the 14 cases there was a predominance of female children with a percentage of $57 \%$ of cases.

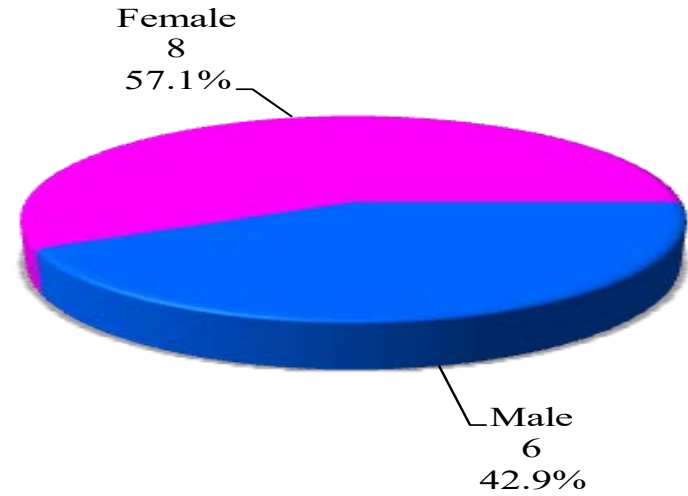

Figure 20: Distribution of the studied cases according to $\operatorname{sex}$

* A questionnaire will be given to the surgeon including the following points:

1) Cost of the fenestrated splint and the cost of the oropharyngeal airway tube.

2) Time consumed during fabrication of the fenestrated splint versus modification of the oropharyngeal airway tube.

3 )Ease of insertion and time during surgery.

4)Fitting of the fenestrated splint versus fitting of the airway tube.

5) Stability of the splint will be recorded as stable if no mobility is present and unstable if mobility of the splint is present.

6) Support of the splint.

7) The need for more modification at the time of surgery.

8) Proper teeth alignment by the splint after reduction and fixation.

Radiographic evaluation and healing of the fracture is monitored after removal of the splint and after 4 months of follow up.

Clinical evaluation of the infant and grading of his comfort with the splint,ability for breastfeeding, maintaining of oral hygiene,and durability of the splint across the 4 weeks are recorded.

Data collection and statistical analysis was done giving results with better total score for the fenestrated splint over the splint made from modification of the oropahryngeal airway tube (Table 1).

Table 14: Comparison between the two studied groups according to total score

\begin{tabular}{|c|c|c|}
\hline Total Score & $\begin{array}{c}\text { Group A } \\
(n=7)\end{array}$ & $\begin{array}{c}\text { Group B } \\
(n=7)\end{array}$ \\
\hline Min. - Max. & $32.0-37.0$ & $22.0-31.0$ \\
\hline Mean \pm SD. & $34.0 \pm 2.08$ & $26.43 \pm 3.15$ \\
\hline Median & 34.0 & 26.0 \\
\hline$p$ & \multicolumn{2}{|c|}{$<0.001^{*}$} \\
\hline
\end{tabular}

$\mathrm{p}$ : $\mathrm{p}$ value for Student t-test

*: Statistically significant at $\mathrm{p} \leq 0.05$ 


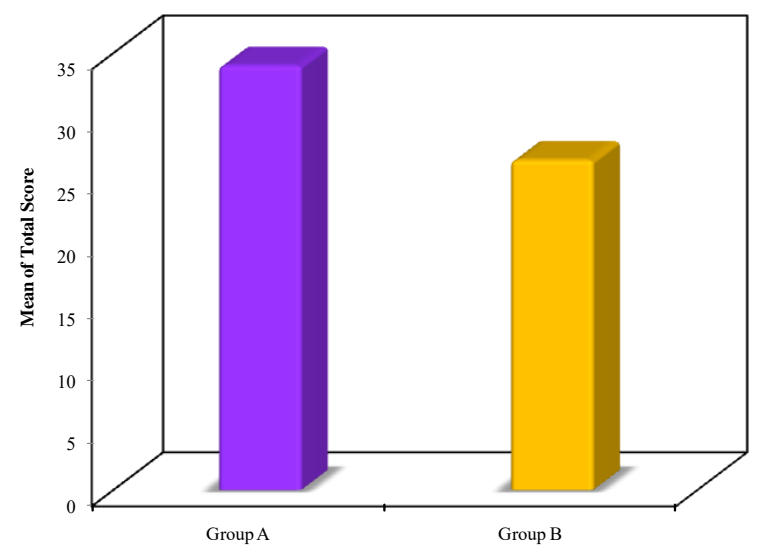

Figure 14: Comparison between the two studied groups according to total score

The fenestrated acrylic splint gives better scores in all points of comparison except in the cost and time required for fabrication of the oropharyngeal airway tube is better because it is cheaper and available in the operating room and doesn't require any special equipments for its construction.

\section{Conclusion}

The mandible is one of the most common sites of facial bone fractures in adults and children.In treating such fractures, the objective is to re-establish pretraumatic function and esthetics of the dentofacial complex with limited morbidity, without hindering future growth and development and without damaging the underlying developing dentition.

The anatomical complexity of the developing mandible and teeth strongly suggest the use of surgical techniques that are very different from those routinely used in adults. The conservative approach is recommended. Whenever possible closed reduction should be the treatment of choice.

Therefore, a splint with circummandibular wiring is the treatment of choice.

In our institution the use of the modified oropharyngeal airway tube to be used as a splint was the treatment of choice for these cases as the oropharyngeal airway tube is always available in the operating room with different sizes and its elasticity makes it easy to be trimmed and modified according to each case.

During our study, the shape of the arch of several cases didn't fit with the corresponding size of the oropharyngeal airway tube, so these cases were shifted to group A to be treated with the fenestrated splint which is easily constructed by the heat cured acrylic resin with good dimensional stability, less residual monomer and few porosities to maintain health of the gingiva and avoid its irritation.

The fenestrated splint is custom made for each case so it's easily inserted in place, fitted around the few erupted teeth with good adaptation and durability all the period of fixation.

The fenestrated splint prepared before surgery reduced time expended by the surgeon before in the preparation and modification of the oropharyngeal airway tube and many surgeons reported that they preferred the use of the fenestrated splint over the modified oropharyngeal airway tube for treatment of mandibular fracture in infants.

On the other hand the modified airway tube is elastic and didn't withstand the forces of occlusion exerted on it during the period of fixation and two cases returned with fractured splint after two weeks of fixation.

The concavity inside the modified oropharyngeal airway tube helped in the accumulation of food debris and bad oral hygiene for group B cases as it is not well adapted on the mandibular arch.

In our study, no case of mandibular fracture due to child abuse was found. Some authors have reported similar cases.

\section{Future Scope}

Splints used for stabilization and fixation of pediatric mandibular body fracture are a reliable and noninvasive procedure that dentists could consider in selected cases through referral to an oral and maxillofacial surgeon. A splint limits the discomfort and morbidity that may be associated with maxillomandibular fixation or open reduction and internal fixation in pediatric patients.

New trends can be used for splint fabrication as CAD/CAM technology and 3D printing with no need for alginate impression avoiding more stresses on the traumatized child, fracture reduction is done virtually on a virtual mandible which would be easier, faster with knowing the exact location of nerves and teeth buds to allow positioning of the fixation wires precisely and avoid any damage to the vital structures.

\section{Conflicts of Interest}

The authors have none to declare.

\section{References}

[1] MacLennan WD. Fractures of the mandible in children under the age of six years. Br J PlastSurg 1956; 9: 125132.

[2] Rowe NL. Fractures of the jaws in children.J Oral Surg 1969; 27: 497-507.

[3] Thoren H, Iizuka T, Hallikainen D. Different patterns of mandibular fractures in children: an analysis of 220 fractures in 157 patients. J CraniomaxillofacSurg 1992; 20: 292-6.

[4] Dufresne CR. Pediatric facial trauma. In: McCarthy JG, editor. Plastic Surgery. 2"d ed. Philadelphia: WB Saunders; 1990. P. 312-44.

[5] Hardt N, Gottsauner A. The treatment of mandibular frac-tures in children.J CraniomaxillofacSurg 1993; 21: 214-9.

[6] Kahan LB, Mulliken JB, Murray JE. Facial fractures in children: an analysis of 122 fractures in 109 patients. PlastReconstrSurg 1977; 59: 15-20.

\section{Volume 5 Issue 7, July 2016




\section{International Journal of Science and Research (IJSR) \\ ISSN (Online): 2319-7064}

Index Copernicus Value (2013): 6.14 | Impact Factor (2015): 6.391

[7] Posnick JC, Wells M, Pron GE. Pediatric facial fractures: evolving patterns of treatment. J Oral MaxillofacSurg 1993; 51: 836-44.

[8] Abbas I, Ali K, Mirza YB. Spectrum of mandibular fractures at a tertiary care dental hospital in Lahore. J Ayub Med Coll 2003; 15: 12-14.

[9] Defabianis P. TMJ fractures in children and adolescents: treatment guidelines. J ClinPediatr Dent 2003; 27: 191-9.

[10] Lustmann J, Milhem I: Mandibular fractures in infants. Reviewof the literature and report of seven cases. J Oral Maxillofacsutg 52:240, 1994

[11] GooddayRH.Management of Fractures of the Mandibular Body and Symphysis. Oral Maxillofacial SurgClin N Am 2013;25: 601-616

[12] http://dx.doi.org/10.1016/j.coms.2013.07.002

[13] Singh M, et al. Management of pediatric mandibular fractures using bioresorbable plating system - Efficacy, stability, and clinical outcomes: Our experiences and literature review, J Oral BiolCraniofac Res. 2015;189, http://dx.doi.org/10.1016/j. jobcr.2015.09.004

[14] PosnickJc.Mandibular Fractures in Infants: Review ofthe Literature and Report of Seven Cases.J Oral MaxillofacSurg52245-246, 1994

[15] Swaify GA etal.A versatile splint for fractured mandible in infants.the Saudi Dental Journal 1991; 3:72_74.

\section{Author Profile}

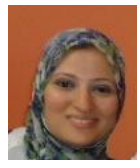

Dr. Yomna Ahmed El Shazly is BDS faculty of dentistry Alexandria University

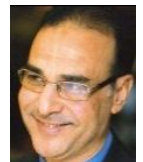

Prof. Dr. Ahmad Mohammed Alshimy is Prosthodontic department Faculty of dentistry, Alexandria University

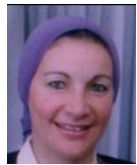

Prof. Dr. EnayaAlySharara is Prosthodontic department, Faculty of dentistry, Alexandria University

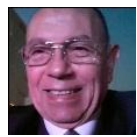

Prof. Dr. GamalAlyElswaify Oral and maxillofacial Department, Alexandria University 